\title{
Book review on cancer research and therapy: Safety concerns for HPV vaccination of young generation, Paid by Obamacare and V.P. Biden Moonshot initiative
}

\author{
Mahin Khatami* \\ Inflammation and Cancer Biology, National Cancer Institute (Ret), the National Institutes of Health, Bethesda, MD 20817, USA
}

\begin{abstract}
In a recent multidisciplinary book on evaluation of cancer research and therapy for a century, the author provides overwhelming evidence on scientific basis, heavy politics and hidden agenda behind the high failure rates $(90 \%+/-5)$ of cancer therapy in the last century. Evidence is presented on creation of a highly ordered and sophisticated medical hierarchy with motives to profit from sale of drugs, particularly in USA. The power of establishment grew since 1955 when public was inoculated with virus-contaminated polio vaccines despite the warning of a competent scientist who discovered that vaccines were contaminated with SV 40 viruses! In the last six decades the incidence and mortality of cancer sharply increased. The current baby boomers also suffer from numerous other chronic diseases such as autoimmune and neurodegenerative diseases and require consumption of drugs that often cause adverse or life-threatening side effects and need more drugs. American health status is at the bottom of 17 or 11 other developed nations although USA invests highest amount of funds for healthcare.

For the first time evidence was presented that the highly ordered power structure of cancer/medical establishment verses total lack of order in cancer research and therapy and claims of 'targeted' therapies, 'precision' or 'personalized' medicine is potent recipe for repeated failed outcomes that kill patients but generates huge corporate profits. Conducting too many out-of-focus expensive projects on identification of endless somatic mutations that are basis for drug development are 'scientific ponzi schemes' or 'molecular false flags' on false foundations. This report provides outlines of reported cancer research and therapy of the last century with emphasis on scientific concerns about long-term safety for HPV vaccination of young generation that could potentially create another wave of diseases for next baby boomer' generation to be drug-dependent. The motives behind vaccinating the young generation with HPV or meningitis vaccines are the availability of insurance through Affordable Care (Obamacare) and additional support of VP Biden Moonshot Initiative for preparation, advertisement, recruitment and vaccination of unsuspecting public.

Answer to cancer and increased public health is possible only if policy makers and cancer-stricken public realize that the might of power over the right of science must be drastically changed and reversed. Otherwise, 'if you don't know, or don't want to know, how to use resources, they will never be enough'. Developing universal vaccines requires systematic understanding of natural ability of body to fight foreign elements or effective immunity and maintenance of balance between two highly regulated and biologically opposing arms that we defined as Yin (tumoricidal) and Yang (tumorigenic) of acute inflammation. The policy makers in the Congress and the decision makers who direct biological sciences should return to the forgotten value of common sense and logics that our Forefathers used to serve the public. Otherwise, we may be intelligent, but if not able to think and love well being of others, we use the intelligent against humanity.
\end{abstract}

\section{Introduction}

In a multidisciplinary book, the author described formation of a highly ordered and sophisticated medical hierarchy (medical establishment) in late 19th and early 20th century within higher education institutions (e.g., medical schools) for health education with the support of businessmen and philanthropists with motives to profit from sale of drug, particularly in the United States of America [1]. The power of establishment grew since 1955 when public was intentionally inoculated with million doses of virus-contaminated polio vaccines that sharply increased the deadly cancer incidence and mortality and numerous chronic diseases such as autoimmune and neurodegenerative diseases of the current 'baby boomer' generation in America. Inoculation of public proceeded despite the warning by a competent scientist at NIH who discovered that polio vaccines were contaminated with SV 40 viruses! In the last six decades cancer incidence and mortality has sharply increased, particularly in America. Numerous other disabling acute or chronic illnesses [e.g., poliomyelitis, vasculitis, autoimmune and neurodegenerative diseases or vaccine-associated paralytic polio (VAPP)] have been reported as the results of public vaccination with contaminated polio vaccines. The abusive power, unethical behaviors and criminal activities of the establishment were further enhanced since 1971, when the Cancer Act, signed by President Nixon, increased the cancer research funding of National Cancer Institute (NCI)/NIH to $1.6 \mathrm{~B}$, so that the cancer problem is solved in 8 years. In the last four decades, the scam in cancer research and treatment and reductionist approaches to therapy directed and enforced by members of the establishment who are

Correspondence to: Mahin Khatami, Inflammation and Cancer Biology, National Cancer Institute (Ret), the National Institutes of Health, Bethesda, MD 20817, USA, Tel: 301-469-0111; E-mail: MKGoodness@aol.com

Key words: cancer establishment, Moonshot Initiative, affordable care, Obamacare, $H P V$ vaccines, polio vaccines, baby boomer generation, American health status, financial toxicity, safety, Yin-Yang acute inflammation, hypersensitivity, viruses, bacteria, genetic mutations, targeted therapy, precision or personalized medicine, molecular false flags, cancer tsunami

Received: September 29, 2016; Accepted: October 13, 2016; Published: October 17, 2016 
in charge of public funds and projects to make huge profits for the Cancer Corporation at the cost of public lives. The establishment has been successful in collecting/spending several trillions of dollars ( $\$ 1.6$ trillion was spent in 2008 alone on cancer drugs and care) with the claims of 'cancer cure' and care. The war on cancer has been a huge enterprise and a very expensive Government Welfare Program for the members of the establishment who enjoy career longevities of 40-65 years and obtaining continued large sums of grants, often with little serious review processes. Members of establishment and their handlers within and outside NCI/NIH, Big pharma and selected individuals/ institutions around the world, receive professional recognitions and excessive travel expense accounts whether or not the projects produce anything of value to benefit the society [1-5].

To assess the extent of influence of contaminated polio vaccines around the world and at the request of World Health Organization (WHO), a multidisciplinary epidemiologic project was formed. As detailed in the book, in 2005 the report concluded that all vaccines produced in the USSR were heavily infected with SV40 until at least 1978 and with the exception of the Italian vaccines, which were contaminated until 1999, those vaccines that were prepared in the Western world have been SV40-free since 1963. However, analyses of other data are controversial whether the 'vaccines that were prepared in the Western world have been SV40-free since 1963. Therefore, for more than a decade public were exposed to virus-contaminated vaccines, the influence of which is that current baby boomer and their offspring are sicker than the generation before, although modern medicine and the existence of antibiotics and technologies and improved hygiene prolonged average age [1].

It is also alarmingly disappointing that while over 20 million publications exist on cancer-related topics, the scientists still argue whether inflammation is protective or destructive to the body. The author believes that the reductionist approaches and the creation of a double vision for the mission of cancer by the decision makers (establishment, insiders) in cancer community who emphasis and fund too many projects that are claimed as 'targeted' therapies, 'personalized' or 'precision' medicine, are the principal reasons for extremely slow progress and repeated failed outcomes [1-5].

As detailed in a recent publication, the medical/cancer establishment is a huge monopoly composed of various governmental agencies (e.g., $\mathrm{NCI} / \mathrm{NIH}, \mathrm{FDA}, \mathrm{CDC}$ ), and numerous private or public institution and corporate components (e.g., academia, cancer centers, Big pharma, food industry) within and outside the USA forming a corporatization of corruption [1]. Collectively, the establishment directly and indirectly controls the health of population in USA and around the globe. The establishment has the sum characteristics of corporate greed, bribe, criminal and unethical actions (corruption) that are reported for other governmental agencies or private organizations and financial institutions. However, the cancer establishment has a unique and dangerous feature for criminal activities that cannot be independently verified or easily challenged by policy makers or professionals who benefit from maintaining their silence. Members of the cancer/medical establishment who are in charge of projects (professionals with high scientific recognitions and awards, including Nobel prize) are licensed to make the public sick and drug-dependent or kill patients under the mission of public health and with banners of 'war on cancer', 'Stand Up to Cancer' or 'cancer cure'. The establishment is entitled to promote, glamorize and publicize vaccination of the public with little/ no considerations for long-term health effects or consumption of too many drugs that are often unnecessary, harmful or life-threatening with little challenge from independent professionals [1-5].

In summary, despite investing excessive amount of American resources for public health, and according to official reports American health ranks 47 th out of 50 ; the bottom 11th from eleven or the last of 17 other developed countries.

In this report principle reasons for lack of progress in cancer research and therapy will be outlined with emphasis on safety concerns and motives that establishment is urging and targeting the young generation to be vaccinated with HPV or meningitis vaccines. Abuse of funds and availability of additional support through V.P. Biden Moonshot Initiative and Affordable (Obamacare) insurance and philanthropists are major incentives for heavily publicity to vaccinate the public without any ethical considerations for long-term health consequences of such visionless projects. Potential creation of next generation of 'Baby Boomer' to become a sick society that is drugdependent is outlined below. Future logical and systematic directions for cancer research and therapy and development of universal vaccine to promote immunity are briefly discussed [1-9].

\section{Forgotten Common Sense and Logics in Science: Fun- damental Discoveries and Theories verses Lack of Log- ics and Reductionist Approaches to Cancer Research and Control: A Double Vision in Mission of Cancer!}

Details of fundamental and historical observations and theories of Rudolph Virchow, Paul Ehrlich, Peyton Rous or Sir McFarland Burnet with regard to important role of immunity in control of cancer cells are described in recent publications [1-5]. In particular, the immune surveillance theory that Burnet articulated in a 1957 publication is an important theory that provides scientists a logical foundation for understanding the mechanisms of immunity in protecting the body against external or internal molecular or cellular defects or insults such as pathogens, biological, chemical or environmental hazards or carcinogens that threaten the survival of the body throughout life. However, systematic extension and application of these fundamental observations, discoveries and theories for cancer research and therapy have been practically ignored and downplayed by the decision makers in the cancer community for a century [1]. Instead, the decision makers created a reductionist approach to cancer research and therapy for motives that is far from conducting cost effective and logical projects to benefit the society and prevent, promote health or treat cancer and other age-associated chronic diseases. The heavy investments of public and private funds are disproportionately used for identification of endless genetic mutations in the chaotic molecular environments of site-specific cancers. The results of reductionist approaches in targeting one or combination of dysfunctional molecules [e.g., proteins, enzymes, metabolites, or factors that are identified as apoptotic (causing growtharrest/necrosis/tumoricidal) or growth factors (growth-promote/ tumorigenic)] in the chaotic molecular tsunami of site-specific cancer produced failure rates of $90 \%(+/-5)$ while patients suffer from the life-threatening side-effect of cancer drugs (poisons) and pay for their treatments that postpone their death-sentence for short durations.

These approaches that repeatedly have failed are 'molecular false flags' and are based on false foundations. However, these approaches that are claimed 'targeted' therapies, 'personalized' or 'precision' medicine continue to be heavily popularized by the decision makers under the banners of 'cancer cure' or 'Stand Up to Cancer' to collect more money from the cancer-stricken public. 
Since the sharp increase in cancer incidence and mortality, careers of professionals in multidisciplinary biomedical fields of microbiology and infectious diseases and cancer research have been secured. Countless funded projects support investigations on details of SV-40 or other viruses or endless genetic and protein molecular species within the chaotic cancer environment for finding a 'cure' for cancer. However, little/no interest has been demonstrated to follow the historical observations and theories on the role of immune system in control of cancer cells, to systematically understand stimuli(e.g., viruses, bacteria, antigens, carcinogens)-induced early immune response dynamics that initiate loss of body's effective immunity for controlling and fighting external or internal elements such as cancerous and other defective cells that threaten the body's health.

With the availability of modern technologies, the academicians and 'intellectuals' in cancer biology have become so narrowly experts in their fields of research (e.g., virology, genetics, proteomics, lipidomics, metabolomics) that they know the details of molecular structures and substructures of viruses or site-specific tumors. However, cancer remains an imaginary problem that is 'too difficult' to solve and the establishment continue to demand more funding to discover more molecules with no light at the end of the tunnel! Furthermore, while the cascades of cancer metastasis to secondary specific organs are relatively understood ( 1 , manuscript in preparation), systematic studies of multistep in carcinogenesis that are the most important events for understanding the loss of cancer control mechanisms, which could lead to effective detection and prevention as well as, therapy remain as a mystery.

These 'specialists' whose careers are secured from continuous funding of such reductionist approaches remind us the spiritual statement of Rumi; that-'People cannot see the camel in the minaret but they can see the hair in its nose'! [1].

Therefore, a major factor in the extreme slow progress for understanding cancer biology is total disregard and absence of systematic studies to understand the initial molecular events that lead to altered immune response profiles of vulnerable tissues toward multistep carcinogenesis. For example, except for the 'accidental' discoveries that our research team at University of Pennsylvania established in 1980 's on experimental models of acute and chronic inflammation, there is no other evidence on early stages of immune dysfunction that would lead to multistep tumorigenesis and angiogenesis. The results of these fundamental studies provided the first series of evidence for a direct link between inflammation and tumorigenesis. Re-analyses of the original data demonstrated the first evidence on sequential interactions and synergies between host immune and non-immune cells and those of recruiting inflammatory cells during developmental phases of immune dysfunction in the direction of cell growth in ocular tissues. The author describes that her submitted logical concepts, comprehensive proposals and related lectures including proposed use of anti-inflammatory drugs that were extension of her original discoveries, initially met with severe opposition, denial and rejection by decision makers and their handlers at NCI upper management since 1998. However, the hijacked ideas were fragmented and used by members of the establishment as fronts to collect more money from the cancer-stricken public in the last decade. Unfortunately, ongoing debates, misinformation and misunderstanding of the role of inflammation, whether it is protective in preventing cancer or it is a cause of cancer have been very costly to the public, including the recent reductionist approaches for immunotherapy that repeatedly failed.
Lack of oversight and accountability on abuse of funds on too many worthless projects that repeatedly failed made cancer an imaginary difficult problem that cannot be solved!

In summary, the cancer establishment has been very successful in creating and glamorizing a false foundation for applying fraudulent science in treating patients with drugs (poisons) that postpone their death sentence for short durations while their resources (insurance and personal assets) are drained for going through torturous periods of repeated visits to physician offices, hospitals and tests. The claimed 'targeted' therapies, 'personalized' or 'precision' medicine that are based on identification of endless somatic mutations in the chaotic cancer molecular environments (cancer molecular tsunami) are 'molecular false flags' that are based on false foundations (Figure 1).

The molecules that are identified and isolated and reported in the literature by specific and selective technologies from different laboratories include over-, or under-expression of growth or apoptotic factors, cytokines/chemokines, dysfunctional or defective (activated or inactivated) enzymes, receptor molecules, DNA/RNA/miRNAs, mitochondrial bioenergetics (mitophagy), ribosomal protein/lipid recycling pathways [autophagy] or dysfunctional molecules that are derivatives of the disrupted biophysical and mechanical architectural integrities in cancerous tissues.

Such incredibly fraud approaches to cancer research and therapy which repeatedly failed the patients remind us of finding too many pieces of many broken needles in a haystack and attempting to put them back together. Loss of patients lives (particularly the politicians and celebrities loved ones) seem to become incentives for the establishment with its world's largest lobbying group to go before the Congress and public to provide attractive versions of the same lip services on how 'remarkable' achievements have been but they need 'more money' to continue.

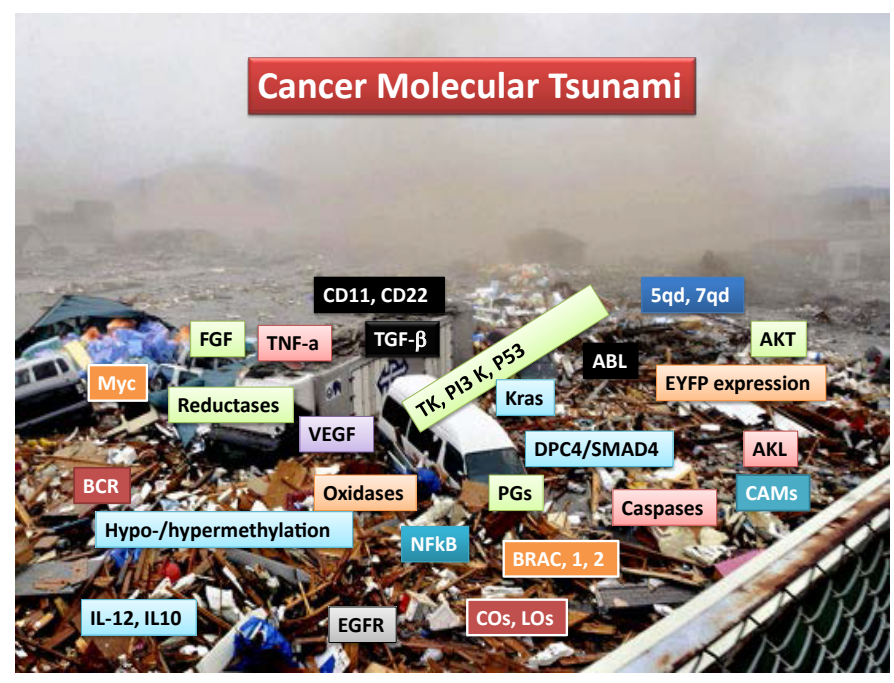

Figure 1. 'Cancer Molecular Tsunami'. The picture resembles the severely disrupted integrities of molecular, cellular and sub-cellular structures of immune and non-immune systems in site-specific cancers. Many candidates for drug development are numerous defective molecules, genetic mutations, over-, under-, or co-expression of growth and apoptotic factors, cell surface molecules, receptor molecules, decoy receptors and cytokines/ chemokines, enzymes/proteins or vascular and membrane components [e.g., p53, ALK, AKT, NFkB, PI3K, MAPKs, Myc, Hsp-90, VEGF, IGF, FGF, IFN- $\gamma$, TNFdR, MMPs, CRP, S1P, CD44, CD73, CD146, CD166, CD90, CD105/CD1-5, PTEN, TGF- $\beta$, PDL-1, IL2, IL10, COs, Los, TLRs, mTOR, caspases, tryptase, chymase, oxidases, PGE2] derived from dysfunctional immune and non-immune cells activities. Photo shows the 2011 Japanese Tsunami. Source: Internet. Reproduced from the book (Reference 1), all rights reserved. 


\section{Safety Concerns and Political Motives for Targeting Young American Population for HPV Vaccination Funded by Obamacare and VP Biden Moonshot Initiative. Potential Creation of another Sick and Drug- Dependent Society:}

While data on the role of virus-causing cancers existed half a century prior to injecting the American population with million doses of virus-contaminated polio vaccines in 1955, the decision makers in the establishment downplayed and ignored the existing data including the serious safety concerns and warnings of a competent microbiologist at $\mathrm{NIH}$ who discovered that polio vaccines were contaminated [1]. In this article, the author raises serious concerns that the American tragedy that damaged the health of current baby boomers half a century ago could be repeated, if the young population is inoculated with HPV or other vaccines (e.g., meningitis). This is particularly important because the young population born from parents (one or two generations ago) who were exposed to contaminated vaccines, may have higher risks for diseases. Several reports demonstrate the increased incidence of allergies and other immune or metabolic diseases in the last few decades.

This article is timely because additional resources through wellintentions of policy makers and philanthropists (e.g., V.P. Biden Moonshot Initiative, Obamacare, Bill and Melinda Gates Foundation and countless other small or large donations) are likely to end up supporting the reductionist approaches of the same medical/cancer establishment that created the cancer tragedy for the current 'baby boomers' to be drug-dependent. Serious scientific concerns are raised that HPV vaccination of the young generation which is being heavily publicized and supported by additional funding, is another visionless scheme of decision makers at $\mathrm{NCI} / \mathrm{NIH}$ and BigPharma, for huge corporate profits. It seems that the Affordable Care Act (Obamacare) which provides healthcare funding for millions of Americas youth has been a huge incentive for the establishment to target the young population for HPV and meningitis vaccines and maintain total control of public health young and old!

The safety concerns of the heavily advertised vaccines against human papilloma viruses (HPV) with the claims to prevent cervical and other cancers should be taken seriously and before it is too late. A summary of scientific concerns about long-term safety of HPV vaccination of the young generation is provided below. Detailed biological consequences that HPV vaccination could potentially create another wave of diseases for the next 'baby boomer' generation to be drug-dependent are provided in author's recent book [1].

Human papilloma viruses (HPVs) are small heterogeneous family of over 130 different viruses (HPV types) of double-stranded DNA whose potencies and genomic structures and substructures evolve from time to time, from individual to individual and from tissue to tissue. A wide range of HPVs have been identified in organs/tissues such as the skin, larynx, vagina, penis, esophagus, conjunctiva, bronchus, paranasal sinuses, tracheo-bronchial and oral mucosa, anogenital tract, urethra, and in such human diseases as the genital warts, recurrent respiratory papillomatosis, low-grade and high-grade squamous intraepithelial lesions (SILs), as well as, anal, vaginal and cervical cancers [1].

It is important to emphasize that while a great deal of public investment has been devoted for identifying details of chemical, biological and genomic structures and substructures of viruses, bacteria or parasites (microorganisms) or numerous carcinogens including expression products and mechanisms of actions of the evolving characteristics of such microorganisms (e.g., HPV, polio, herpes, AIDS, EBOLA, influenza, measles, hepatitis A, B, LPS, meningitis or Zika) that often behave differently in different susceptible tissues and from individual to individual to cause a wide range of infections, neurological or metabolic disorders or cancers, there is a peculiar absence of study to understand what initiates the immune disruptor,(virus, bacterium, carcinogen)-induced altered biological properties of susceptible tissues that would lead to manifestation of virus-causing multistep diseases and site-specific cancers.

The author argues that emphases on production of specific vaccines to inactivate/kill or prevent one or combination of HPVs DNA segments/ entities/activities and/or their expression products, while not effective to prevent specific diseases (e.g., cervical cancers), the long-term of HPV vaccines could contribute to initiation of other health problems during adulthood and aging. The genomic structures of the HPV viruses of vaccines (e.g., inactivated high potency viral components) could shift to activate other compensating mechanisms (e.g., mutations of viral DNA components that are not as virulent) to survive and evolve to different forms of virus (virus-like-particles), to ensure survival of viruses' dormant life ('low risk') in host's multilayered organ systems and cause health hazards as soon as they found opportunity to escape the host immunity. A wide range of vaccine-related complications including autism (measles vaccines), multiple sclerosis (hepatitis B vaccines), menangioencephalitis (Japanese encephalitis vaccines), Guillian-Barre syndrome and giant cell arthritis (influenza vaccines), encephalomyelitis (semple rabies vaccines) or neurological problems (e.g., H1N1 or Swine flu vaccines) demonstrated the harmful effects of such pathogen-specific vaccines. Analyses of relevant data on adverse health effects of polio or other vaccines suggest the possibility of integration of viral or bacterial DNA into host tissue affect the dynamics of immunity from different dimensions. In addition to the reported adverse effects of polio vaccines, the health hazards of other vaccines indicate that introduction of segmented viruses, bacteria or parasites (inactivated pathogens or antigenic components of microorganisms) to the body are not tolerated by body's immunity [1].

Like cancerous cells, viral or bacterial oncogenes and substructure particles are viewed as internal or external foreign entities to be neutralized or ignored by effective immunity as long as they co-exist in the multilayered cellular organization of host organ systems. However, loss of effective immunity provides opportunities for these pathogens to be activated and take advantage of host compromised immunity for their survival and growth and/or cause/induce integration and transformation to host chromosomal components.

We further hypothesized that the exposures to viral-containing vaccines, or inhibiting/inactivating specific high risks or 'potent' segments of viruses' DNA (e.g., HPVs) impact the homeostasis and dynamics (ecosystem) of the host microorganisms (e.g., GI track, skin), particularly in young adults. In later life altering the hazard/benefit ratios of body's microbiota may be important contributing factors in the induction of chronic diseases such as allergies, autoimmune or neurodegenerative complications or cancers, as demonstrated for other infectious diseases.

The young generation in America is already vulnerable to diseases, evidenced by increased allergies and metabolic disorders that are perhaps due to the genetic predisposition of baby boomer generation and consumption of virus-contaminated vaccines of the past generation (s) 
It was suggested that conducting such undertakings for public health, require serious scientific logics and right decisions to avoid and minimize risks of diseases. A universal vaccine, or a prophylactic candidate that would generally enhance or stabilize the innate immune cells (e.g., mast cells, dendritic cells, natural killer cells and/ or macrophages) to also influence the resting capability of adaptive immune cells (e.g., $\mathrm{T}$ and $\mathrm{B} /$ plasma cells) and crosstalk with nonimmune systems (e.g., vasculature, neuronal and metabolic pathways) for boosting the body's natural effective immunity should be the focus of future studies for preventing diseases. However, a universal vaccine development is a challenging and huge undertaking, particularly because after 2 centuries of investing trillions of dollars and over 20 million publications on cancer related research and therapy, we know very little on the early molecular events that lead to alterations of response dynamics and initiation of immune dysfunction toward genesis of diseases. The microorganisms-induced early alterations in immune dynamics are very likely reversible, preventable or correctable/ druggable.

\section{Concluding remarks}

For more than a century, through formulated laws the directors of institutions at NIH or other governmental health agencies (FDA, CDC, DHHS) and nearly all heads of cancer centers, medical schools, Big Pharma or food industry (who introduced the genetically modified organisms/GMOs to the public) are MDs (physicians). The only formal mission and duty of these leaders should have been improving and promoting public health, preventing diseases and saving the lives of the Americans. However, despite investing excessive amount of American resources for public health the opposite has occurred. According to governmental and private organizations reports the American health ranks 47 th out of 50; the bottom 11th from eleven, or the last of 17 other developed countries.

For the first time the author describes that creation of the highly sophisticated power structure in the cancer/medical establishment versus absence of any organization or system to study cancer are hidden agenda behind high failure rates of $90 \%(+/-5)$ of cancer drugs that generated a financial empire to use patients as experimental models for testing drugs at the patients' expense (insurance and personal assets) and lives. The new schemes for vaccinating the young generation that is supported by Affordable care and Moonshot Initiative and other private funding are visionless approaches and could generate another sick society that is drug-dependent. It is projected that provision of extra funds for vaccination of young generation will not produce anything of value for promoting health, except it could jeopardize the health of future generation who are the new target of the cancer/medical establishment and the makers of vaccines. We should have learned something from the Cancer Act of President Nixon, four decade ago when the same empty promises were made to solve cancer problem.

Answer to cancer and increased public health is possible only if policy makers and the cancer-stricken public realize that the might of power over the right of science must be drastically changed and reversed. The author's recent publication is a wake-up call to make sure that the evil part of human being does not prevent the health services that the public deserves. Otherwise, it does not matter how many resources you have, if you don't know, or don't want to know, how to use them, they will never be enough'.

Systematic understanding of the effective immunity of host and its protective mechanisms against all internal and external foreign entities, and how to promote the organ systems defense ability, particularly in aging process, have greater priorities than investing resources on understanding the details of evolving genomic substructures of trillions of viruses for producing vaccines.

The major goals for future research directions should be focusing on promotion of immune defense system provided through the Yin (tumoricidal) and Yang (tumorigenic) properties of acute inflammation for monitoring, controlling and arresting growth of cancer cells and preventing age-associated diseases. Such studies require systematic understanding of what initiates cancer cells to evade immunity and takeover the machinery of the host for their survival that ultimately destroy their host. In such studies it is necessary to understand the important roles that mitochondria play not only as a energy source for maintenance of Yin-Yang, but also for mitochondria-dependent biosynthesis of structural proteins that are required for architectural integrity of cells, prosper anchoring and contact inhibition, properties of tissues that are impaired to varying degrees in age-associated chronic diseases including site-specific tissues [1-6].

We recently hypothesized that cancer is a severe and cumulative delayed type hypersensitivity responses of site-specific tissues. Histamine at low circulating level is a blueprint that maintains oxidative stress and differentially contributes to the genesis and progression of tissue necrosis or growth manifested as neurodegenerative or sitespecific cancers. The author describes that histamine being an alkaline and vasoactive agent may contribute to the dysfunction of cellular components, including mitochondrial function and acid-base balance of membrane components [2].

Finally, the policy makers in the Congress and the decision makers who direct biological sciences should bring back the forgotten common sense and logics that our Forefathers used to serve the public. Otherwise, we may be intelligent, but if not able to think and love well being of others, we use the intelligent against humanity.

\section{Acknowledgement}

The author's experimental studies were established on experimental models of acute and chronic ocular inflammatory diseases, at the University of Pennsylvania, with a support team of scientists headed by John H. Rockey in 1980's. Analyses of original data that resulted in a series of 'accidental' discoveries on the role of inflammation in time-course kinetics of altered immune dynamics toward multistep carcinogenesis and angiogenesis were extended at the National Cancer Institute (NCI)/NIH since 1998 and despite heavy opposition, denial and rejection of submitted proposals by members of cancer establishment.

\section{References}

1. Khatami M (2016) In Cancer Research and Therapy: Scam of Century-Promote Immunity [Yin-Yang]. 1-166. [Crossref]

2. Khatami M (2016) Cancer a severe delayed hypersensitivity and histamine a blueprint? Working model to promote Yin and Yang of immunity. Clin Transl Med 5: 35. [Crossref]

3. Khatami M (2014) Chronic inflammation: synergistic interactions of recruiting macrophages (TAMs) eosinophils (Eos) with host mast cells (MCs) and tumorigenesisun CALTs. MCSF, suitable biomarker for cancerdiagnosis! Cancers (Basel) 6: 297-322. [Crossref]

4. Khatami M (2012) Unresolved inflammation and cancer: loss of natural immune surveillance as the correct 'target' for therapy! Seeing the 'Elephant' in the light of logic.Cell Biochem Biophys 62: 501-509. [Crossref]

5. Khatami M (2011) Unresolved inflammation: 'Immune tsunami' or erosion of integrity in immune-privileged and immune-responsive tissues and acute and chronic inflammatory diseases or cancer. Expert Opin Biol Ther 11: 1419-1432. [Crossref] 
Khatami M (2016) Book review on cancer research and therapy: Safety concerns for HPV vaccination of young generation, Paid by Obamacare and V.P. Biden Moonshot initiative

6. Khatami M (2008) "Yin and Yang" in inflammation: duality in innate immune cell function and tumorigenesis. Expert Opin Biol Ther 8: 1461-1472. [Crossref]

7. Khatami M (2007) Standardizing cancer biomarkers criteria:data elements as foundation for a database. Inflammatory mediators/M-CSF as model marker. Cell BiochemBiophys. 47: 187-198. [Crossref]

8. Khatami M (2005) Developmental phases of inflammation-induced massive lymphoid hyperplasia and extensive changes in epithelium in an experimental model of allergy: implications for a direct link between inflammation and carcinogenesis. Am J Ther 12: 117-126. [Crossref]

9. Khatami M (2005) Cyclooxygenase inhibitor ketorolac or mast cell stabilizers: immunological challenges in cancer therapy. Clin Cancer Res 11: 1349-1351. [Crossref]

10. Khatami M (2012) Inflammation, aging and cancer: Friend or foe? In: Khatami M (ed) Inflammation, chronic diseases and cancer-cell and molecular biology, immunology and clinical bases. InTech Publishing 3-30. [Crossref]

11. Khatami M, Donnelly JJ, John T, Rockey JH (1984) Vernal conjunctivitis. Model studies in guinea pigs immunized topically with fluoresceinylovalbumin. Arch Ophthalmol 102: 1683-1688. [Crossref]

12. Khatami M, Donnelly JJ, Haldar JP, Rockey JH (1989) Massive follicular lymphoid hyperplasia in experimental chronic recurrent allergic conjunctivitis. Arch Ophthalmol 107: 433-438. [Crossref]

Copyright: $(02016$ Khatami M. This is an open-access article distributed under the terms of the Creative Commons Attribution License, which permits unrestricted use, distribution, and reproduction in any medium, provided the original author and source are credited. 